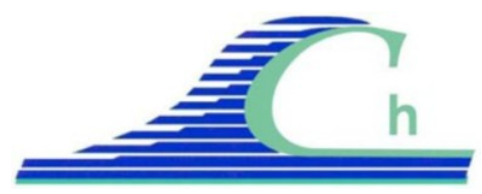

XII ${ }^{\text {èmes }}$ Journées Nationales Génie Côtier - Génie Civil

Cherbourg, 12-14 juin 2012

DOI:10.5150/jngcgc.2012.002-C C Editions Paralia CFL

disponible en ligne - http://www.paralia.fr-available online

\title{
Impact du changement climatique sur les vagues et les flux sédimentaires le long du littoral aquitain
}

\section{Elodie CHARLES ${ }^{1}$, Déborah IDIER ${ }^{1}$, Pascale DELECLUSE ${ }^{2}$, Michel DEQUE ${ }^{3}$, Gonéri LE COZANNET ${ }^{1}$}

1. BRGM, 4 av Claude Guillemin, 45060 Orléans, France. elodie.charles29@gmail.com;d.idier@brgm.fr;g.lecozannet@brgm.fr

2. Météo-France / Centre National de Recherches Météorologiques, 73 avenue de Paris, 94165 Saint-Mandé Cedex, France. pascale.delecluse@meteo.fr

3. Météo-France / Centre National de Recherches Météorologiques, 42 avenue Gaspard Coriolis, 31057 Toulouse Cedex, France.

michel.deque@meteo.fr

\section{Résumé :}

La dynamique sédimentaire littorale est contrôlée par un ensemble de facteurs hydrodynamiques qui sont susceptibles d'être modifiés par le changement climatique. Les vagues ont un rôle dominant dans la dynamique du littoral aquitain. Afin d'analyser l'impact du changement climatique, un modèle de vagues est mis en place et des simulations sont réalisées pour les climats actuel (1961-2000) et futurs (2061-2100). L'analyse des futures conditions de vague dans le Golfe de Gascogne et le long de la côte aquitaine a mis en évidence une diminution généralisée des hauteurs de vague et une rotation horaire de l'ensemble des vagues estivales et des houles hivernales par rapport au climat actuel. Ces changements résulteraient en une diminution des flux de sédiments longshore au niveau de la plage de Biscarrosse, de l'ordre de $10 \%$.

Mots-clés: Vagues - Changement climatique - Transport sédimentaire - Côte aquitaine - Modélisation

\section{Introduction}

Le changement climatique est susceptible de modifier les caractéristiques actuelles du littoral. Bordée par le Golfe de Gascogne, la côte aquitaine est principalement composée de plages sableuses. Dans ce secteur, les vagues ont un rôle prédominant dans la dynamique sédimentaire du littoral (CASTELLE et al., 2007). Les analyses existantes des futures conditions de vagues portent principalement sur les hauteurs de vagues à l'échelle mondiale et prédisent soit une variation non significative des hauteurs de vagues, soit une diminution (DEBERNARD \& ROED, 2008 ; ZACHARIOUDAKI et $a l ., 2011)$. Elles sont basées sur des projections de vagues présentant une résolution spatiale faible et indiquent des résultats très variables dans le Golfe de Gascogne. De plus, l'étude de la dynamique littorale nécessite d'autres paramètres que la hauteur de 
vagues, à savoir les périodes et directions de vague. Une étude plus régionale des conditions de vague futures a donc été réalisée afin d'évaluer dans un premier temps les changements potentiels des conditions de vagues dans le Golfe de Gascogne et d'analyser dans un second temps l'évolution des flux de sédiments longshore liés aux vagues le long de la côte aquitaine. Cette étude se base sur des projections de vagues réalisées pour le Golfe de Gascogne, présentant une résolution spatio-temporelle adaptée à l'analyse des conditions de vagues du large à la côte (jusqu'à une profondeur d'environ $20 \mathrm{~m}$ ).

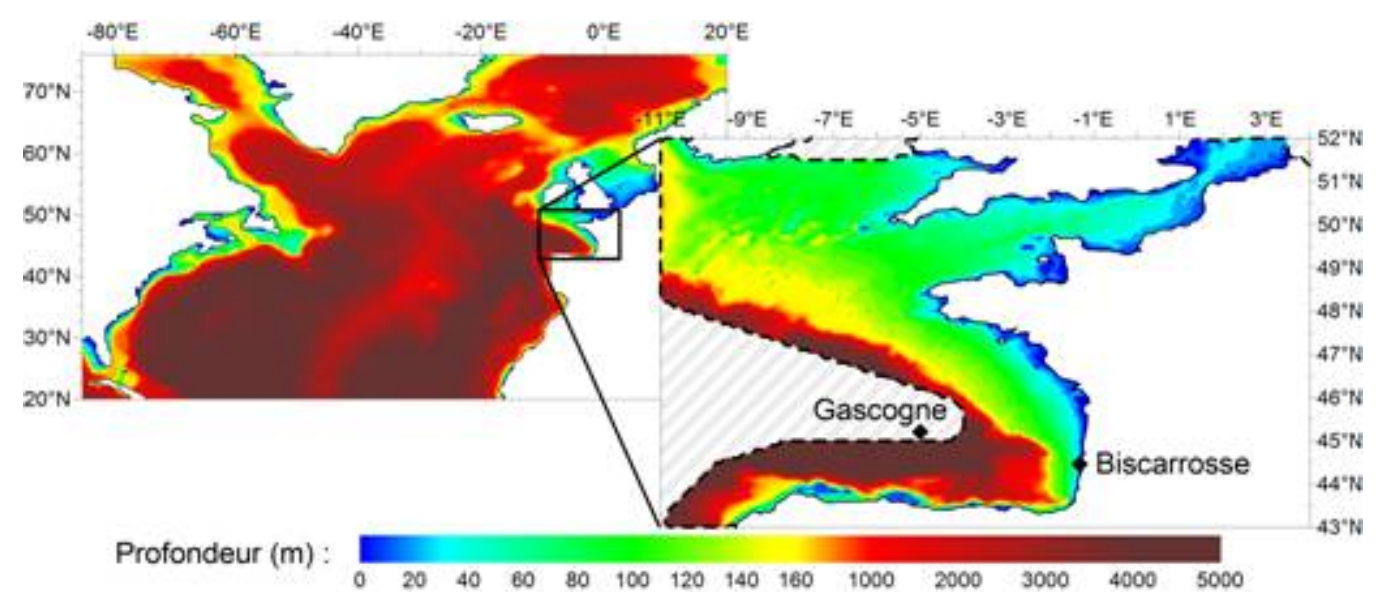

Figure 1. Domaines utilisés pour modéliser les vagues avec WW3. Les zones hachurées (profondeurs supérieures à $4000 \mathrm{~m}$ ) sont exclues du second domaine. Les positions des bouées Gascogne et Biscarrosse sont indiquées par des diamants.

\section{Méthode et données}

Les conditions de vague dans le Golfe de Gascogne et le long de la côte aquitaine sont issues de la base de données BoBWA-10kF (Bay of Biscay Wave Atlas - Forecast, avec une résolution spatiale de $10 \mathrm{~km}$, CHARLES et al., 2012b). Les hauteurs, périodes et directions des vagues sont disponibles pour un scénario de référence (REF) présentant les mêmes concentrations de gaz à effet de serre et d'aérosols que celles observées sur la période 1961-2000 et pour trois scénarios futurs (A2, A1B et B1) sur la période 20612100. Les vagues issues de cette base de données ont été simulées avec le modèle spectral de vagues WAVEWATCH III (WW3, TOLMAN 2009), avec le paramétrage d'ARDHUIN et al. (2010). Les vagues ont été modélisées sur deux domaines emboîtés (figure 1), l'Atlantique Nord $\left(\mathrm{dx}=0.5^{\circ}\right)$ et le Golfe de Gascogne $\left(\mathrm{dx}=0.1^{\circ}\right)$, à partir de champs de vent projetés par le modèle ARPEGE-Climat (GIBELIN \& DEQUE, 2003). Une correction de type quantile-quantile a été réalisée sur les hauteurs, périodes et directions de vague, afin de corriger les biais systématiques du modèle climatique. Cette correction a été construite en comparant les distributions statistiques des vagues issues de BoBWA-10kF-REF à celles des vagues issues de BoBWA-10kH (Bay of Biscay 


\section{XII ${ }^{\text {èmes }}$ Journées Nationales Génie Côtier - Génie Civil \\ Cherbourg, 12-14 juin 2012}

Wave Atlas - Hindcast, avec une résolution spatiale de $10 \mathrm{~km}$, CHARLES et al., 2012a). Dans la suite de cette étude, nous utilisons les paramètres corrigés de hauteur significative (Hs), de période moyenne (Tm-10) et de direction moyenne (Dm) issues de BoBWA-10kF, sur le domaine couvrant le Golfe de Gascogne $\left(0.1^{\circ} \mathrm{x} 0.1^{\circ}\right.$, toutes les 6 heures) et au niveau des bouées Gascogne $\left(5^{\circ} \mathrm{W}-45.201^{\circ} \mathrm{N}\right.$, profondeur : $4500 \mathrm{~m}$, issue du rang 1) et Biscarrosse $\left(1.32^{\circ} \mathrm{W}-44.46^{\circ} \mathrm{N}\right.$, profondeur : $\left.26 \mathrm{~m}\right)$ (toutes les heures).
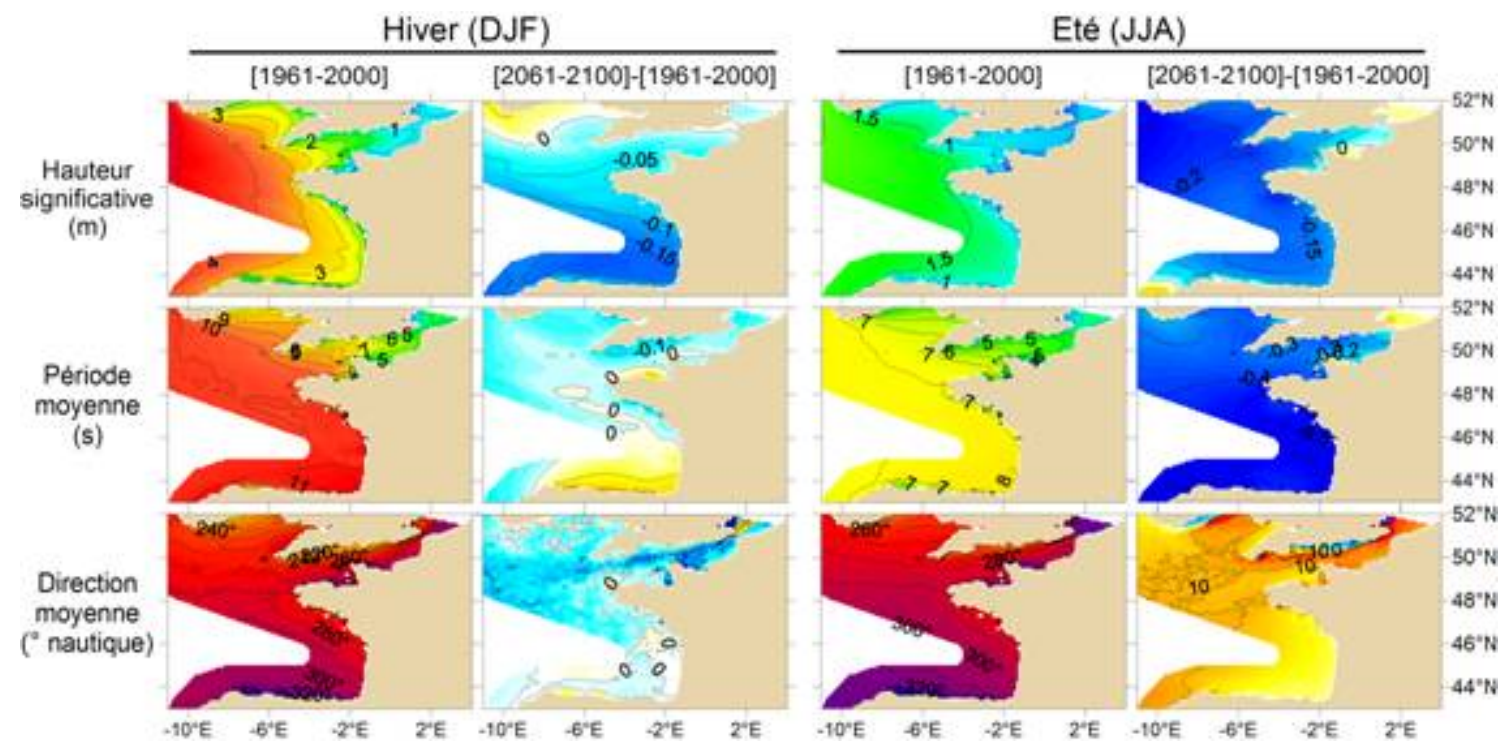

Figure 2. Cartes des hauteurs, périodes et directions moyennes pour le scénario présent

REF (colonnes 1 et 3), et différences entre le scénario futur A2 (2061-210) et le scénario présent REF (1961-2000) (colonnes 2 et 4) pour l'hiver et l'été. (adapté de

CHARLES et al., 2012b).

\section{Analyse du climat de vagues futur}

Les changements des conditions de vagues dans le Golfe de Gascogne et le long de la côte aquitaine sont analysés pour l’hiver (déc., jan., fév.) et l'été (juin, juil., août). On s'intéresse en particulier au scénario A2, qui présente un taux d'émissions de gaz à effet de serre plus élevé que les scénarios A1B et B1, et donc des changements plus marqués par rapport au climat actuel. Les conditions de vague du scénario REF sur la période 1961-2000 sont comparées aux conditions de vague du scénario A2 sur la période 20612100. Dans un premier temps, les changements sont analysés à l'échelle régionale, sur le domaine du Golfe de Gascogne, puis dans un second temps à l'échelle locale, au niveau d'une bouée hauturière et d'une bouée côtière.

A l'échelle régionale (figure 2), on peut noter les changements suivants dans le Golfe de Gascogne : une diminution généralisée des hauteurs de vague (jusqu’à -20 cm en été), une rotation horaire des directions de vague en été (de $3^{\circ}$ à $10^{\circ}$ ) et une légère diminution des périodes en été (environ -0.5 s). 
Ces changements moyens sont la somme des changements de plusieurs types de vagues (houle, mer de vent, vagues intermédiaires). Afin de mieux caractériser leur évolution, une étude approfondie est réalisée en deux points : au niveau de la bouée Gascogne, située au centre du Golfe de Gascogne, à $4500 \mathrm{~m}$ de profondeur, et au niveau de la bouée Biscarrosse, située le long de la côte aquitaine, à $26 \mathrm{~m}$ de profondeur. Les diagrammes bivariés (figure 3) des conditions de vagues pour le climat présent (scénario REF, 1961-2000) permettent de visualiser les variations du climat de vagues suivant la saison et suivant la distance à la côte. En hiver, les vagues sont plus énergétiques qu'en été (fortes hauteurs et périodes de vague). Au niveau de la bouée côtière Biscarrosse, les effets bathymétriques induisent une réduction des hauteurs de vagues et un resserrement des directions autour de la normale à la côte.

Tableau 1. Méthode choisie de répartition des vagues en fonction de leur cambrure ( $c=2 \pi H_{s} / g T_{m}^{2}$ avec $\rho$ la densité de l'eau, Tm la période moyenne, g l'accélération de pesanteur et Hs la hauteur de vague significative) ou de leur flux d'énergie ( $\left.Q=\rho T_{m} g^{2} H_{s}^{2} / 64 \pi\right)$. Pour plus de détails, voir (CHARLES et al., 2012a). L'indice 50 indique la valeur médiane annuelle et $c_{P M}$ correspond à la cambrure de PiersonMoskovitz $\left(c_{P M}=1 / 19.7\right)$.

\begin{tabular}{ll}
\hline Cambrure & Type de vagues \\
\hline$c<c_{50}$ & Houle \\
$c_{P M}<c<c_{50}$ & Vagues intermédiaires \\
$c \approx c_{P M}$ & Mer de vent \\
\hline
\end{tabular}

\begin{tabular}{ll}
\hline Flux d'énergie & Type de vagues \\
\hline$Q<Q_{50}$ & Vagues peu énergétiques \\
$Q>Q_{50}$ & Vagues énergétiques \\
\hline
\end{tabular}

Concernant les changements entre les conditions de vagues du climat futur (scénario A2, 2061-2100) et du climat présent (scénario REF, 1961-2000), on note une évolution différente en été et en hiver (figure 3). Les valeurs médianes de la cambrure c, du flux d'énergie $\mathrm{Q}$ et de la direction Dm sont calculées à partir des données annuelles de REF au niveau de chaque bouée et sont reportées sur les diagrammes bivariés afin d'en faciliter l'analyse. Le tableau 1 résume la méthode et la terminologie choisies pour désigner les vagues en fonction de leur cambrure ou de leur flux d’énergie. En hiver, au niveau des deux bouées, l'occurrence de la houle augmente, tandis que l'occurrence des vagues intermédiaires diminue. En hiver, au niveau de la bouée Gascogne, les vagues dont la hauteur est supérieure à $3 \mathrm{~m}$ présentent une rotation dans le sens horaire (figure 3). Cette rotation n'est pas visible au niveau de la bouée Biscarrosse. En été, au niveau des deux bouées, l'occurrence des vagues énergétiques diminue, et l'occurrence des vagues peu énergétiques augmente. Les vagues dont la hauteur est inférieure à $3 \mathrm{~m}$ présentent une forte rotation horaire. Cette rotation est moins prononcée au niveau de la bouée côtière. 


\section{XII èmes Journées Nationales Génie Côtier-Génie Civil \\ Cherbourg, 12-14 juin 2012}
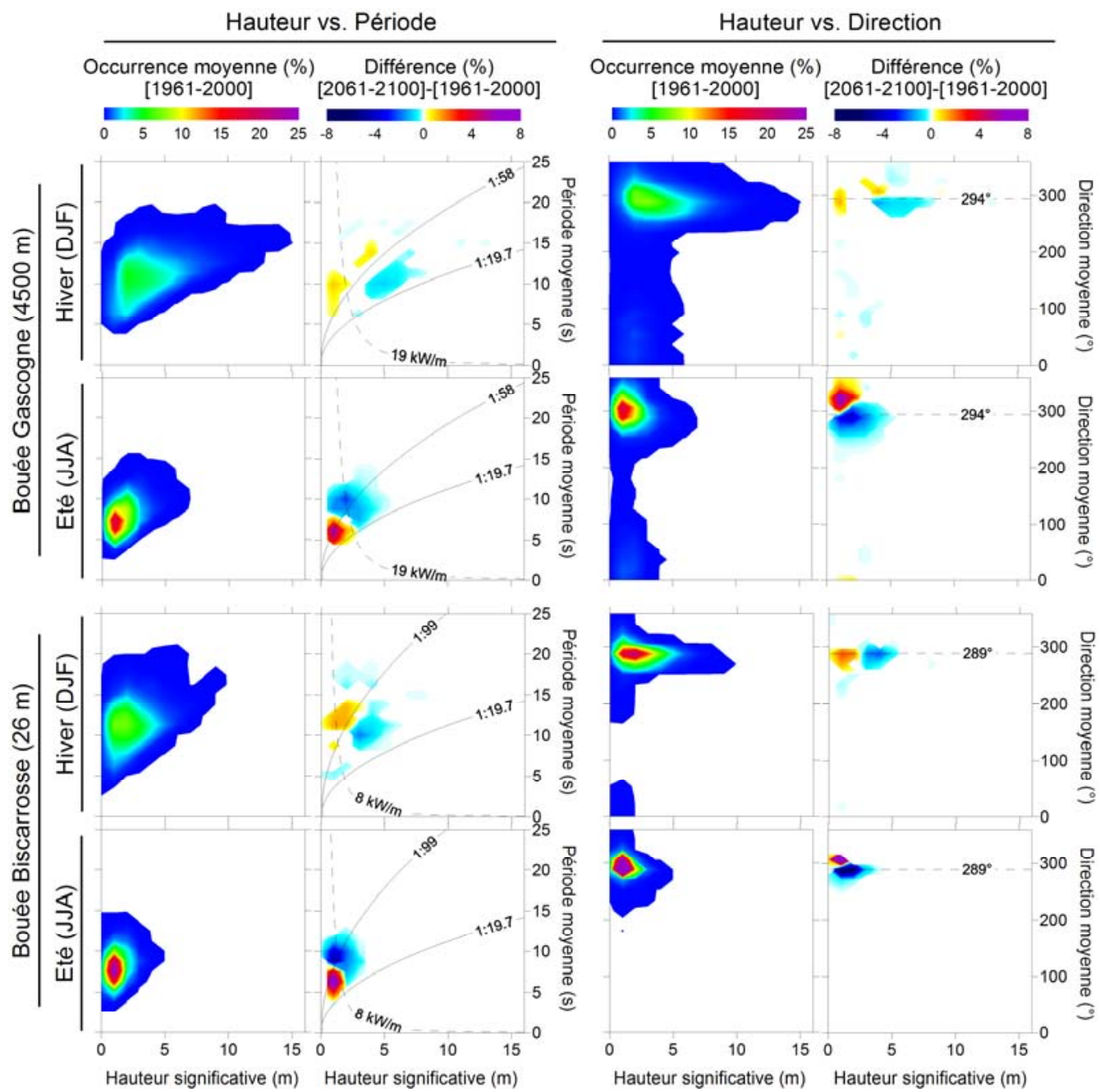

Figure 3. Diagrammes bivariés des conditions de vagues au niveau des bouées Gascogne (haut ; adapté de CHARLES et al., 2012b) et Biscarrosse (bas) pour le scénario présent REF et diagrammes bivariés des changements entre le scénario futur A2 (2061-2100) et le scénario présent REF (1961-2000) pour l'hiver et l'été. Seuls les changements significatifs à plus de 95\% (T-Test de Student) sont tracés. Dans la $2^{e}$ colonne, les lignes continues correspondent à la cambrure médiane annuelle et à la cambrure de Pierson-Moskovitz, la ligne pointillée correspond à la valeur médiane annuelle du flux d'énergie. Dans la $4^{e}$ colonne, la ligne continue correspond à la direction médiane annuelle.

Ces changements de conditions de vagues locales sont produits par des changements de conditions de vent à différentes échelles spatiales. L'analyse détaillée des relations entre changements de vents et de vagues a été réalisée par ailleurs (CHARLES et al., 2012b). Considérons ici par exemple la rotation horaire des directions de vagues observée l'été. A l'échelle locale, une rotation horaire des directions de vent génère un changement de direction de la mer de vent. A l'échelle régionale, il existe aussi une rotation horaire des 
directions de vent au Nord et à l'Ouest du Golfe de Gascogne (les vents étant davantage orientés vers le Sud pour le climat futur), impliquant que plus de vagues se propagent du Nord vers le Sud, et donc entrent dans le Golfe de Gascogne par la frontière Nord et moins par la frontière Ouest. Enfin, à l'échelle océanique, on note une diminution des vitesses de vent dans certaines zones de l'Atlantique Nord, engendrant une réduction de l'occurrence des houles d'Ouest et d'Ouest-Nord-Ouest.

\section{Impact des changements de conditions de vagues sur les flux sédimentaires longshore}

Il est possible d'estimer les ordres de grandeur des flux longshore de sédiments à partir des conditions des vagues. En particulier plus les hauteurs de vagues sont fortes et/ou plus l'angle d'incidence des vagues est fort (en restant inférieur à 45², CERC, 1984), plus les flux sont forts. Or, l'analyse précédente montre que les hauteurs et directions des vagues au large sont affectées par le changement climatique. En outre, les résultats des simulations montrent que la diminution des hauteurs de vague identifiée au large entre climat présent et climat futur se propage jusqu'à la côte, tout comme les changements de direction affectant les petites vagues, bien qu'ils soient atténués par la réfraction bathymétrique. Ainsi, dans cette partie, nous analysons dans quelle mesure ces changements pourraient affecter de manière significative les flux longshore à l'horizon 2100.

Une analyse de l'évolution temporelle des flux de sédiments longshore a été réalisée pour la plage de Biscarrosse, en utilisant les conditions de vagues simulées au niveau de la bouée Biscarrosse. Plusieurs formulations permettent d'estimer les ordres de grandeur de ces flux à partir des conditions de vague. Globalement, elles dépendent toutes explicitement de la hauteur de vagues et de leur incidence. Certaines, comme celle de KAMPHUIS (1991) intègrent aussi explicitement la pente de la plage, la taille de grain, ou la période des vagues. Cependant, comme rappelé par PILKEY and COOPER (2002) il existe une forte incertitude dans les résultats obtenus à partir de formules de transport longshore, et, au mieux, seuls les ordres de grandeur sont à considérer.

Dans un premier temps, une estimation préliminaire de l'ordre de grandeur des flux absolus est effectuée en utilisant les formules du LCHF (1979) et de KAMPHUIS (1991). En effet, ce sont des formules qui apparaissent adaptées entre autres aux côtes Atlantique aquitaine et portugaises (CAYOCCA, 1996 ; ABADIE et al., 2006 ; BALOUIN et al., 2005). Ces formules utilisent les conditions de vague au point de déferlement. Celles-ci sont obtenues en utilisant le jeu de données de vagues modélisé au large de Biscarrosse (1961-2000) et en y appliquant les lois de Snell et de conservation d'énergie, et ce, en faisant donc l'hypothèse que pour le cas étudié les contours bathymétriques sont rectilignes et parallèles ainsi qu'en négligeant la dissipation de l'énergie avant le déferlement. Ainsi, les formules du LCHF (1979) et de KAMPHUIS (1991) appliquées à ces données de vague au déferlement donnent des flux 


\section{XII ${ }^{\text {èmes }}$ Journées Nationales Génie Côtier - Génie Civil \\ Cherbourg, 12-14 juin 2012}

de sédiments de l'ordre resp. de 160000 et $200000 \mathrm{~m}^{3} / \mathrm{an}$. On peut noter que ces estimations sont du même ordre de grandeur que celles d'ABADIE et al. (2006) - 100 $000 \mathrm{~m}^{3} / \mathrm{an}$, valeur estimée à environ $40 \mathrm{~km}$ au sud de Biscarrosse - et de LCHF (1987) $520000 \mathrm{~m}^{3}$ /an, valeur estimée à Mimizan. Parmi les pistes à explorer pouvant expliquer les différences obtenues, outre la localisation spatiale exacte, on peut noter que les données de vagues en entrée diffèrent en nature et période couverte, avec un jeu de 40 ans de données modélisées pour la présente étude, 8 ans (1997-2005) de données modélisées (NOAA-WW3) corrigées pour ABADIE et al. (2006), et des données observées sur une période antérieure à 1985 pour les estimations du LCHF (1987). Toutefois, les flux estimés restent du même ordre de grandeur (O(100 $\left.\left.000 \mathrm{~m}^{3} / \mathrm{an}\right)\right)$.

Afin d'étudier la différence entre les flux actuels et les flux futurs, nous analysons les flux principalement en termes de valeur relative, soit par rapport au flux annuel (figure 4.a), soit par rapport au flux du climat actuel correspondant (figure 4.b). Les flux relatifs donnés résultent de la moyenne des valeurs obtenues avec les deux formules, une barre d'erreur indiquant les valeurs minimale et maximale obtenues avec ces formules.

La figure 4.a donne un aperçu de la variabilité saisonnière des flux de sédiments longshore. La quantité de sédiment déplacée est plus importante de $60 \%$ en hiver par rapport à la moyenne annuelle, et deux fois plus petite en été. Les écarts entre les formules sont plus importants pendant ces deux saisons. Au printemps et en automne, les flux ont une amplitude similaire à la moyenne annuelle.

a)

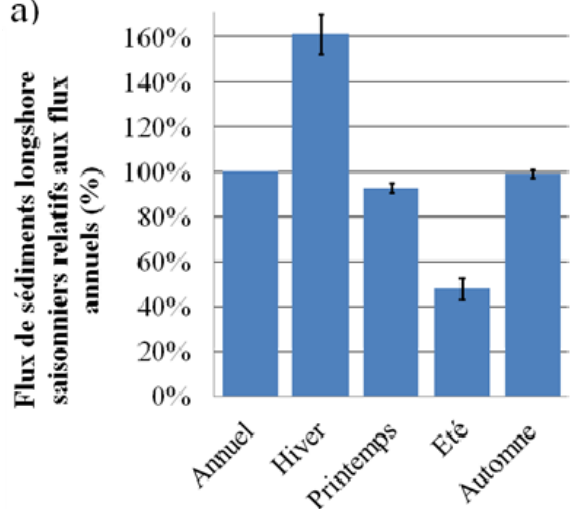

b)

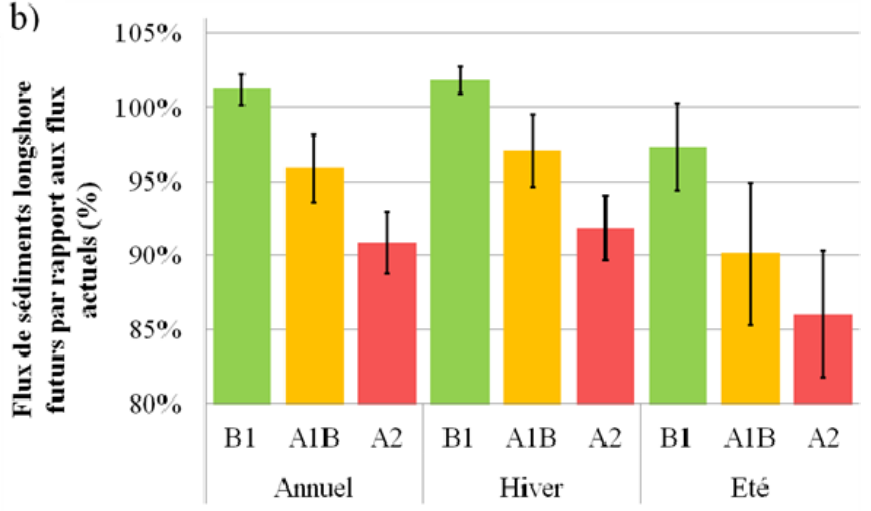

Figure 4. a) Variations saisonnières des flux de sédiments longshore actuels (scénario $R E F)$, relativement au flux annuel, b) Flux de sédiments longshore pour trois scénarios futurs $(B 1, A 1 B, A 2)$, relativement au flux actuel pour la même saison.

Pour le climat futur (figure 4.b), nous nous intéressons en particulier à l'évolution des flux sur l'année, en hiver et en été. Quelle que soit la période de l'année, on constate que les changements sont d'autant plus importants que le scénario est émissif en gaz à effet de serre. Ainsi, les changements de vagues projetés pour le scénario A2 donnent une réduction du flux de l'ordre de $10 \%$ par rapport au climat actuel. Seuls les résultats 
pour le scénario B1 montrent une légère augmentation des flux de sédiments longshore. Ces résultats indiquent que, dans cette région, la baisse projetée des hauteurs de vagues pour le climat futur serait le facteur prédominant dans l'évolution des flux de sédiments. La rotation horaire des vagues projetée, ayant pour effet d'intensifier les flux sédimentaires vers le Sud dans cette région, serait donc largement compensée par la diminution des hauteurs de vague.

Afin de pouvoir analyser l'impact du changement climatique sur la contribution des flux longshore dans l'évolution du trait de côte, il conviendrait d'estimer les flux longshore futurs et actuel tout le long de la côte aquitaine et d'en analyser les variations spatiales.

\section{Conclusions}

Au large, les conditions de vague projetées pour le scénario A2 indiquent une diminution généralisée des hauteurs de vague (de 10 à $20 \mathrm{~cm}$ ) et une rotation horaire de l'ensemble des vagues en été (de 3 à $10^{\circ}$ ) et des houles en hiver. A proximité de la côte, la diminution des hauteurs de vague est toujours significative. Cependant, la réfraction bathymétrique induit une atténuation de la rotation horaire des directions des vagues, en particulier pour les vagues les plus hautes, telles que les houles hivernales.

L'analyse des flux de sédiments longshore au niveau de la plage de Biscarrosse suggèrerait une diminution de l'amplitude des flux le long de ce segment de la côte aquitaine. Ce résultat, obtenu en un point, ne permet pas de conclure à une tendance à l'érosion ou à l'accrétion sur ce segment, l'érosion ou l'accrétion étant liée aux variations spatiales de flux de sédiments. Cependant, ces résultats permettent de conclure que les changements de conditions de vague projetés sont suffisamment significatifs pour modifier les flux longshore du littoral aquitain. Une perspective à ce travail serait d'analyser l'évolution des flux de sédiments longshore sur la côte aquitaine dans son ensemble afin d'analyser l'impact potentiel de ces modifications de flux longshore sur l'évolution du trait de côte à la fin du $21^{\mathrm{e}}$ siècle.

\section{Remerciements}

Ce travail a été réalisé dans le cadre d'une thèse co-encadrée par le BRGM et le CNRM/Météo-France, bénéficiant d'une bourse de thèse du Fonds AXA pour la Recherche. Les auteurs remercient F. Ardhuin, B. Castelle et R. Pedreros pour leurs contributions.

\section{Références bibliographiques}

ABADIE S., BUTEL R., MAURIET S., MORICHON D., DUPUIS H. (2006) Wave climate and longshore drift on the South Aquitaine coast. Continental Shelf Research, Vol. 26, 1924-1939. 


\section{XII èmes Journées Nationales Génie Côtier-Génie Civil \\ Cherbourg, 12-14 juin 2012}

ARDHUIN F., ROGERS E., BABANIN A., FILIPOT J. F., MAGNE R., ROLAND A., WESTHUYSEN A. V. D., QUEFFEULOU P., LEFEVRE J. M., AOUF L., COLLARD F. (2010). Semi-empirical dissipation source functions for ocean waves: Part I, definition, calibration and validation. J. Phys. Oceanogr., Vol. 40, pp 1917-1941. doi:10.1175/2010JPO4324.1

BALOUIN Y., HOWA H., PEDREROS R. and MICHEL D. (2005) Longshore Sediment Movements from Tracers and Models, Praia de Faro, South Portugal. Journal of Coastal Research, Vol. 21(1), pp. 146-156.

CASTELLE B., BONNETON P, DUPUIS H., SENECHAL N. (2007). Double bar beach dynamics on the high-energy meso-macrotidal French Aquitanian coast: A review. Mar. Geol., Vol. 245, pp 141-159. doi:10.1016/j.margeo.2007.06.001

CAYOCCA F. (1996). Modélisation morphodynamique d'une embouchure tidale: application aux passes d'entée du bassin d'Arcachon. Thèse de doctorat, Univ. Bdx 1.

CHARLES E., IDIER D., THIÉBOT J., LE COZANNET G., PEDREROS R., ARDHUIN F., PLANTON S. (2012a). Present wave climate in the Bay of Biscay: spatiotemporal variability and trends from 1958 to 2001. J. Climate, Vol. 25, pp 2020-2035. doi:10.1175/JCLI-D-11-00086.1

CHARLES E., IDIER D., DELECLUSE P., DEQUE M., LE COZANNET G. (2012b). Climate change impact on waves in the Bay of Biscay, France. Ocean Dynamics, 18 p. doi:10.1007/s10236-012-0534-8

CERC (1984). Shore Protection Manual. 4th ed., 2 vols. Technical report, U.S. Army Engineer Waterways Experiment Station. Washington D.C. :U.S. Gov. Printing Office. DEBERNARD J. B., ROED L.P. (2008). Future wind, wave and storm surge climate in the Northern Seas: a revisit. Tellus A, 60(3), pp 427-438. doi:10.1111/j.1600-0870.2008.00312.x GIBELIN A.-L., DEQUE M. (2003). Anthropogenic climate change over the Mediterranean region simulated by a global variable resolution model. Clim. Dynam., Vol. 20, pp 327-339.

KAMPHUIS J. W. (1991). Alongshore sediment transport rate. Journal of Waterway, Port, Coastal, and Ocean Engineering, Vol. 117(6), pp 624-640. doi:10.1061/(ASCE)0733950X(1991)117:6(624)

LCHF (1979). Etude en nature de la côte aquitaine (entre la Pointe de la Grave et l'embouchure de l'Adour). Rapport établi pour la mission interministérielle pour l'aménagement de la côte aquitaine, Laboratoire Central Hydraulique de France.

LCHF (1987). Catalogue sédimentologique des côtes françaises, De la Baie du MontSaint-Michel à la frontière espagnole, partie B. Collection de la DER d'EdF n65, Paris: Eyrolles.

PILKEY O.H., COOPER J.A.G. (2002). Longshore Transport Volumes: A Critical View. J. of Coast. Res., SI 36, pp 572-580 (ICS 2002 Proceedings).

TOLMAN H.L. (2009). User manual and system documentation of WAVEWATCH III version 3.14. Technical Note 276, NOAA / NWS / NCEP / MMAB. 
Thème 1-Hydrodynamique côtière

ZACHARIOUDAKI A., PAN S., SIMMONDS D., MAGAR V., REEVE D. E. (2011). Future wave climate over the west-European shelf seas. Ocean Dynamics, Vol. 6, pp 807-827. doi:10.1007/s10236-011-0395-6 